\title{
Stream-based Machine Learning for Network Security and Anomaly Detection
}

\author{
Pavol Mulinka \\ CTU Czech Technical University in Prague \\ AIT Austrian Institute of Technology \\ pavol.mulinka@fel.cvut.cz
}

\author{
Pedro Casas \\ AIT Austrian Institute of Technology \\ pedro.casas@ait.ac.at
}

\begin{abstract}
Data Stream Machine Learning is rapidly gaining popularity within the network monitoring community as the big data produced by network devices and end-user terminals goes beyond the memory constraints of standard monitoring equipment. Critical network monitoring applications such as the detection of anomalies, network attacks and intrusions, require fast and continuous mechanisms for on-line analysis of data streams. In this paper we consider a stream-based machine learning approach for network security and anomaly detection, applying and evaluating multiple machine learning algorithms in the analysis of continuously evolving network data streams. The continuous evolution of the data stream analysis algorithms coming from the data stream mining domain, as well as the multiple evaluation approaches conceived for benchmarking such kind of algorithms makes it difficult to choose the appropriate machine learning model. Results of the different approaches may significantly differ and it is crucial to determine which approach reflects the algorithm performance the best. We therefore compare and analyze the results from the most recent evaluation approaches for sequential data on commonly used batch-based machine learning algorithms and their corresponding stream-based extensions, for the specific problem of on-line network security and anomaly detection. Similar to our previous findings when dealing with off-line machine learning approaches for network security and anomaly detection, our results suggest that adaptive random forests and stochastic gradient descent models are able to keep up with important concept drifts in the underlying network data streams, by keeping high accuracy with continuous re-training at concept drift detection times.
\end{abstract}

\section{CCS CONCEPTS}

- Computing methodologies $\rightarrow$ Machine learning; • Security and privacy $\rightarrow$ Network security;

\section{KEYWORDS}

Data Stream mining; Machine Learning; Network Attacks; HighDimensional Data.

Permission to make digital or hard copies of all or part of this work for personal or classroom use is granted without fee provided that copies are not made or distributed for profit or commercial advantage and that copies bear this notice and the full citation on the first page. Copyrights for components of this work owned by others than ACM must be honored. Abstracting with credit is permitted. To copy otherwise, or republish, to post on servers or to redistribute to lists, requires prior specific permission and/or a fee. Request permissions from permissions@acm.org.

Big-DAMA'18, August 20, 2018, Budapest, Hungary

(C) 2018 Association for Computing Machinery.

ACM ISBN 978-1-4503-5904-7/18/08 .. \$15.00

https://doi.org/10.1145/3229607.3229612
ACM Reference format:

Pavol Mulinka and Pedro Casas. 2018. Stream-based Machine Learning for Network Security and Anomaly Detection. In Proceedings of ACM SIGCOMM 2018 Workshop on Big Data Analytics and Machine Learning for Data Communication Networks, Budapest, Hungary, August 20, 2018 (Big-DAMA'18), 7 pages.

https://doi.org/10.1145/3229607.3229612

The research leading to these results has been partially funded by the Vienna Science and Technology Fund (WWTF) through project ICT15-129, "BigDAMA".

Pavol Mulinka has been partially supported by the scientific bilateral cooperation grant Aktion Österreich-Tschechien, AÖCZ-Semesterstipendien, ref.num. ICM-2017-08733 and through CTU student grant SGS18/077/OHK3/1T/13.

\section{INTRODUCTION}

Network traffic monitoring and analysis has taken a paramount role to understand the functioning of the Internet, especially to get a broader and clearer visibility of unexpected events. One of the major challenges faced by large-scale network monitoring applications is the processing and analysis of large amounts of heterogeneous and fast network monitoring data. Network monitoring data usually comes in the form of high-speed streams, which need to be rapidly and continuously processed and analyzed. However, detecting and adapting to strong variations in the underlying statistical properties of the modeled data makes learning data stream analysis a very difficult task.

The application of machine learning models to network security and anomaly detection problems has largely increased in the last decade; however, the general approach in the literature still considers the analysis as an off-line learning problem, where models are trained once and then applied to the incoming measurements. A major challenge is how to deal with network monitoring and analysis applications when considering big and fast network measurements. Critical network monitoring applications such as the detection of anomalies and network attacks, require fast mechanisms for on-line analysis of thousands of events per second.

In this paper we consider a stream-based approach for machinelearning-based network security and anomaly detection, using a collection of machine learning algorithms tailored for the analysis of continuously evolving data. Stream machine learning analysis consists of processing a data instance at a time, inspecting it only once, and as such, using a limited amount of memory; stream approaches work in a limited amount of time, and have the advantage of performing a prediction at any point in time during the stream. 
We consider two specific problems within the stream data analytics domain: (i) firstly, we train and evaluate models taking into account the recent history of the observed stream, training in recent fixed-length or adaptive-length batches of data and testing in subsequent observations; (ii) secondly, we build continuous learning models, which take into account the concept drift - i.e., the changes in the underlying statistics of the learning/prediction target, to periodically re-update the machine learning model through on-line learning. We evaluate the performance of the proposed models on the detection of different types of network attacks and anomalies, using real network measurements collected at the WIDE backbone network, relying on the well-known MAWILab dataset for attacks labeling [1].

Similar to our previous findings when dealing with off-line machine learning approaches for network security and anomaly detection, our results suggest that adaptive random forests and stochastic gradient descent models are able to keep up with important concept drifts in the underlying data by keeping high accuracy with continuous re-training at concept drift detection times. We hope these results would motivate a broader adoption of stream-based machine learning models and approaches to network security and anomaly detection in real-time scenarios.

\section{RELATED WORK \& CONTRIBUTIONS}

The application of learning techniques to the problems of network security and anomaly detection is largely extended in the literature. There are a couple of extensive surveys on general domain anomaly detection techniques [2] as well as network anomaly detection $[3,4]$, including machine learning-based approaches. There is a particularly extensive literature in the application of learning-based approaches for automatic traffic analysis and classification. We refer the interested reader to [5] for a detailed survey on the different machine learning techniques commonly applied to network traffic analysis.

We have been recently working on the application of machine learning models to network security and anomaly detection problems [6-9]. In [7] we introduced Big-DAMA, a big data analytics framework for large scale network traffic monitoring and analysis. In [6] we compare the performance of standard, off-line machine learning models for network security in fixed-line networks, further studying more complex and robust models based on ensemble machine learning techniques. Wireless network monitoring using similar techniques is studied in [9]. The knowledge and conclusions extracted from these papers motivated the introduction of GML Learning [8], a generic machine learning model for network measurements analysis, which achieves high accuracy for many different network analysis problems. However, all these approaches consider the off-line analysis of network measurements, in batchmode like analysis.

The specific application of stream machine learning approaches to network security and anomaly detection is by far more limited; a relevant and representative example linked to current study is presented in [10], where authors evaluate stream-based traffic classification approaches based on Hoeffding Adaptive Trees, using the MAWILab dataset [1] and the MOA machine learning toolkit, as we do in current work.
Naturally, the data stream machine learning domain has a long standing tradition and many interesting references are worth mentioning when considering the application and evaluation of stream machine learning models; these cover general problems related to the learning properties for stream-based algorithms [11, 12], the mining and evaluation processes when dealing with massive datasets [13], the identification of model evaluation issues [14] as well as propositions of general frameworks for data streaming [15]. Of particular relevance for stream machine learning model evaluation are the problems of imbalanced classes and concept drift, which are extensively addressed in [16].

It is possible today to extensively apply stream machine learning approaches, based on the availability of multiple publicly available machine learning tools integrating the data stream analytics paradigms. Tools range from toolkits (VFML [17]), libraries (Apache Spark Mlib [18] and Spark ML) to standalone applications (MOA [19], RapidMiner - formerly YALE [20]) and distributed frameworks (SAMOA [21]). In this paper we use in particular the MOA (Massive Online Analysis) framework.

Within the data stream mining domain, the most used evaluation scheme is known as the prequential or interleaved-test-then-train evolution. The idea is very simple, using each instance first to test an initial or bootstrapped model, and then to train or update the corresponding model. Prequential evaluation can be used to measure the accuracy of a model since the start of the evaluation, by keeping in memory all previous history of instances and evaluating the model in each new instance, but it is generally applied using sliding windows or decaying factors, which forgets previously seen instances in the model update process and focuses on those instances in current sliding window. Different from more traditional fold-cross validation, generally used in the evaluation of off-line machine learning models, the weakness of the prequential evaluation is in general its inherent operation on single experiment runs.

In this paper we consider newer and more efficient approaches to evaluate stream machine learning models, considering in particular the ADWIN (ADaptive WINdowing) [22] prequential evaluation of $\mathrm{k}$-fold cross-validated (CV) stream models [23], and the prequential evaluation of the Area Under the ROC curve (AUC) [24]. Building on our previous work on off-line machine learning models for network security [6,7], and working on top of the same real network measurement datasets - MAWILab [1], we study four stream-based machine learning algorithms through ADWIN prequential k-fold $\mathrm{CV}$ and prequential AUC evaluation, comparing their performance to the corresponding off-line versions of the models.

\section{STREAM-BASED MACHINE LEARNING}

In this section, we describe the application of stream-based machine learning models to the detection of different types of network attacks on real network traffic measurements collected at the WIDE backbone network, using the well-known MAWILab dataset for attacks labeling [1]. MAWILab is a public collection of 15-minute network traffic traces captured every day on a backbone link between Japan and the US since 2001. Building on this repository, the MAWILab project uses a combination of four traditional anomaly 
detectors (PCA, KL, Hough, and Gamma, see [1]) to partially label the collected traffic.

Given that we are dealing with continuous data analysis, we firstly evaluate the variation of the statistical properties of the considered dataset, detecting sudden statistical changes or concept drifts. We then study the performance of four popular stream-based machine learning algorithms, including incremental $k$-NN, Hoeffding Adaptive Trees (HAT), Adaptive Random Forests (ARF) and Stochastic Gradient Descent (SGD).

A commonly applied approach for evaluating the performance of stream-based algorithms is to benchmark them against their corresponding off-line, batch implementations. Therefore, we use as baseline the results obtained in our previous work [6-8] for the corresponding batch-based algorithms, including $k$-NN, Hoeffding Tree (HT-batch), Random Forest (RF-batch) and Stochastic Gradient Descent (SGD-batch). We subtract the batch metric results from prequential results to show the performance of stream algorithms in comparison to their batch versions.

We consider two different evaluation strategies: firstly, the dynamically adjusting window-based approach known as ADWIN (ADaptive WINdowing) [22], which maintains a window of variable size containing training samples. The algorithm automatically grows the window when no change is apparent, and shrinks it when the statistical properties of the stream changes. ADWIN automatically adjusts its window size to the optimum balance point between reaction time and small variance; using ADWIN, we evaluate the proposed algorithms following a new strategy to evaluate stream-based algorithms [23], using prequential k-fold cross validation; the strategy is basically an adaptation of cross-validation to the distributed streaming setting, and assumes we have $\mathrm{k}$ different instances of the corresponding model we want to evaluate, running in parallel. Each time a new sample arrives, it is used for testing in one of the k models selected randomly, and then used for training by all the other models. As evaluation metric, we simply take the model detection/classification accuracy (ACC).

Secondly, we also consider the prequential evaluation of the models using the AUC as performance metric, following the very recently introduced approach in [24]. The machine learning community most often uses the AUC statistic for model comparison, which is simple and informative, and provides more reliable comparisons when dealing with imbalanced data. To calculate the AUC, one needs to sort a given dataset and iterate through each observation. Because the sorted order of observations defines the resulting value of AUC, adding a new observation to the dataset forces the procedure to be repeated; as such, AUC cannot be directly computed on data streams, given the associated time and memory requirements. In [24], authors propose an efficient incremental algorithm that uses a sorted tree structure with a sliding window to compute the AUC using constant time and memory. In this case, and different from $\mathrm{ADWIN}$, we consider fix-length windows of different sizes for the prequential evaluation.

\subsection{Dataset description}

The traffic studied in this paper spans two months of packet traces collected in late 2015. From the labeled anomalies and attacks, we focus on a specific group which are detected simultaneously by the

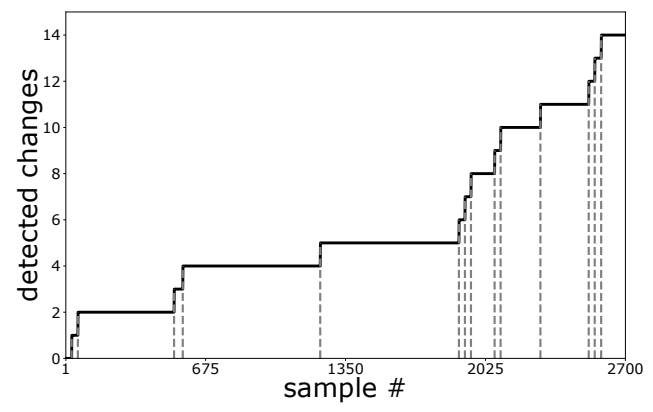

Figure 1: Page-Hinkley Concept Drift Detection. Changes in the dataset distribution detected by the Page-Hinkley test. Detected changes are marked with dashed lines.

four MAWILab detectors, using in particular those events which are labeled as "anomalous" by MAWILab. We consider in particular 5 types of attacks/anomalies: (i) DDoS attacks (DDoS), (2) HTTP flashcrowds (mptp-la), (3) Flooding attacks (Ping flood), and two different flavors of distributed network scans (netscan) using (4) UDP and (5) TCP-ACK probing traffic. We train the different models to detect each of these attack types separately, thus each detection approach consists of five different detectors which run in parallel on top of the data, each of them specialized in detecting one of the five aforementioned attacks types.

To perform the analysis in s stream-based manner, we split the traffic traces in consecutive time slots of five seconds each, and compute a set of features describing the traffic in each of these slots. In addition, each slot $i$ is assigned a label $l_{i}$, consisting of a binary vector $l_{i} \in \mathbb{R}^{5 \times 1}$ which indicates at each position if anomaly of type $j=1 . .5$ is present or not in current time slot. We compute a large number $n=245$ features describing a time slot $i=1 . . m$, using traditional packet measurements including traffic throughput, packet sizes, IP addresses and ports, transport protocols, flags, etc. Note that besides using traditional features such as $\mathrm{min} / \mathrm{avg} / \mathrm{max}$ values of some of the input measurements, we also consider their empirical distribution, sampling the empirical distribution at many different percentiles. This provides as input much richer information, as the complete distribution is taken into account. We also compute the empirical entropy $H(\cdot)$ of these distributions, reflecting the dispersion of the samples in the corresponding time slot. Specific features description is available at [7].

\section{EVALUATION AND DISCUSSION}

In this section we analyze the stream-based algorithms, compare their performance to the corresponding batch-implementations' results and discuss the different evaluation approaches considered in the paper. Results for batch-based implementations correspond to 10 -fold cross validation over the entire dataset. To better understand dynamic changes in the stream-based algorithms performance, we first focus on detecting concept drifts in the studied dataset. Concept drift refers to important changes in the statistical properties of the input data. 


\section{DDoSSYN}
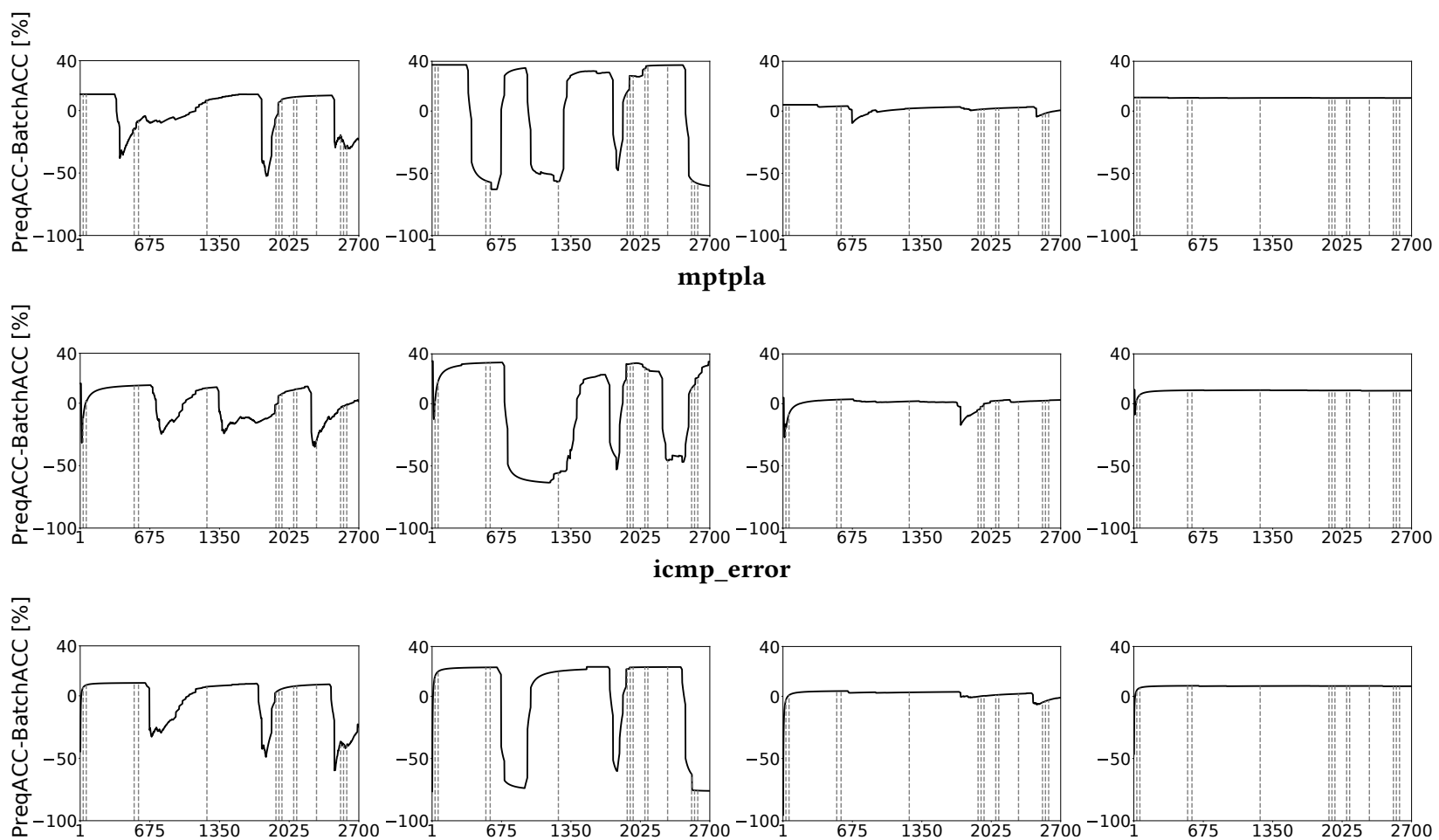

icmp_error
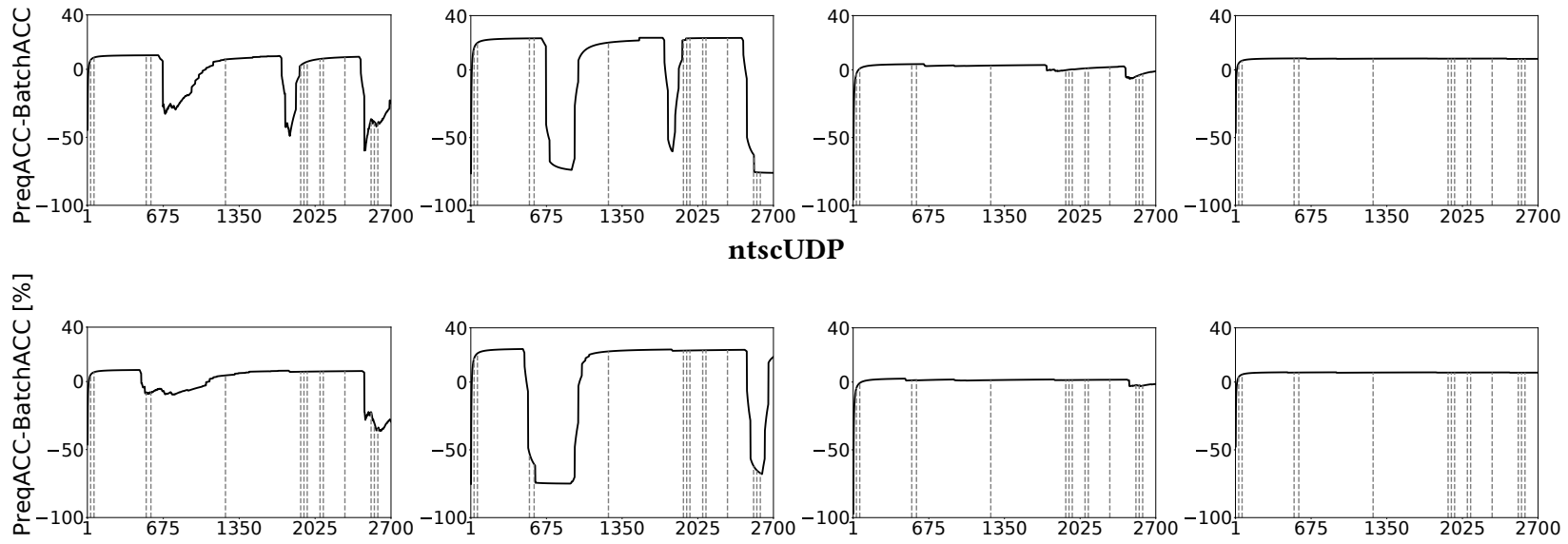

ntscUDP
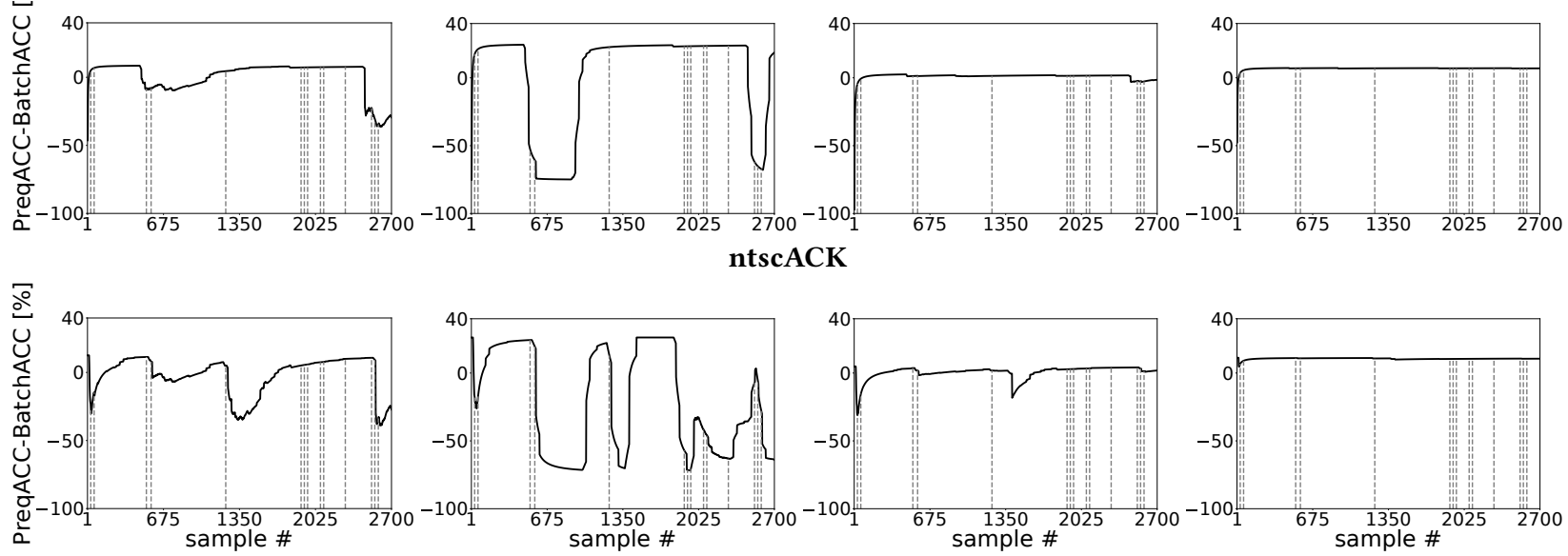

ntscACK

(a) $k-N N$

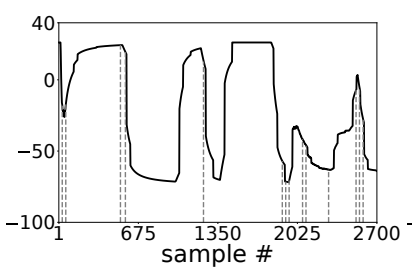

(b) $H A T$

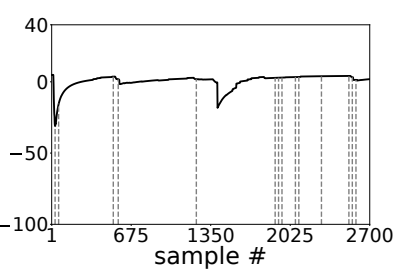

(c) $A R F$

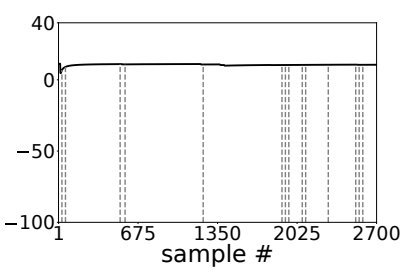

(d) $S G D$

Figure 2: Prequential 10-fold Cross Validation Accuracy Evaluation. Diagrams show Prequential 10-fold CV results for each algorithm for each attack type. Values above zero show when the stream algorithm performance exceeds the batch result. Concept drifts detected by the Page-Hinkley test are marked with dashed lines.

\subsection{Concept Drift Detection}

Concept drift happens when the statistical properties of the analyzed dataset abruptly shift in time [25]. To detect concept drifts, we apply a standard statistical test for change detection known as the Page-Hinkley test, commonly used for change detection in time series analysis. In a nutshell, the test is a sequential adaptation of the detection of an abrupt change of the average of a Gaussian signal, and it allows efficient detection of changes in the normal behavior of a process.

Fig. 1 depicts the cumulative number of changes observed in the dataset, as well as the times when those changes are detected. The test detects 14 abrupt changes during the total measurements time 

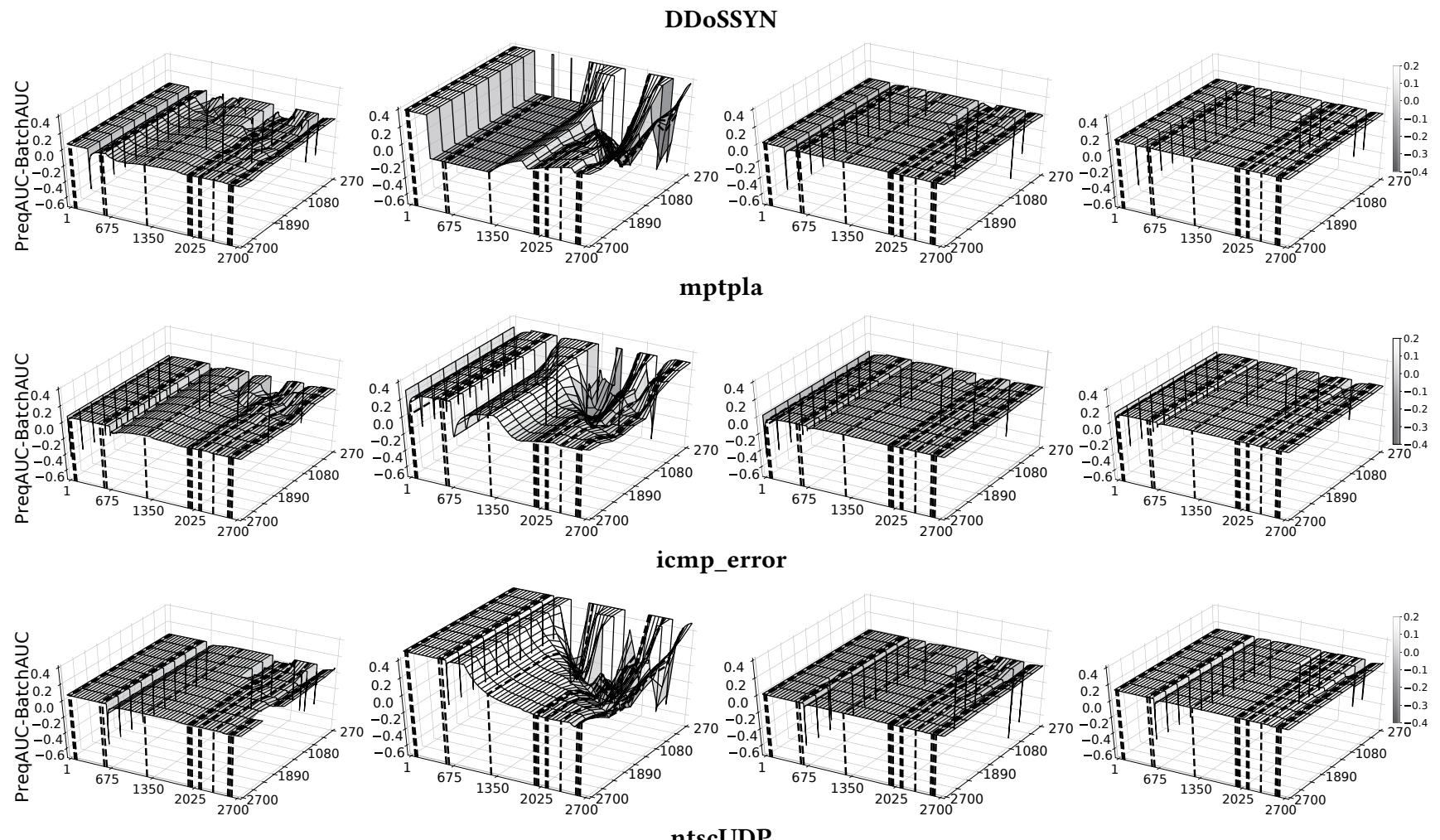

ntscUDP

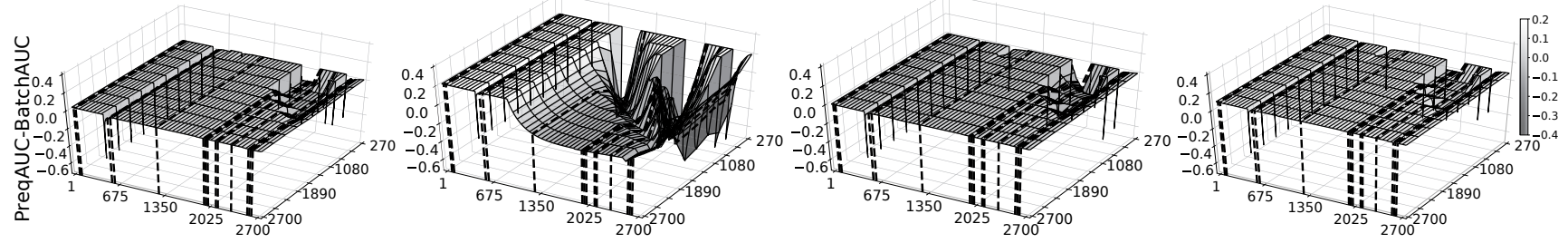

ntscACK

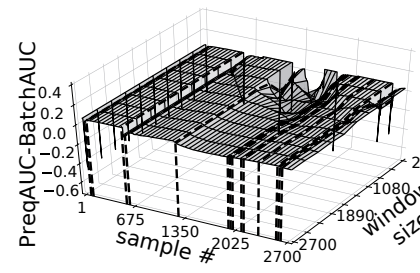

(a) $k-N N$

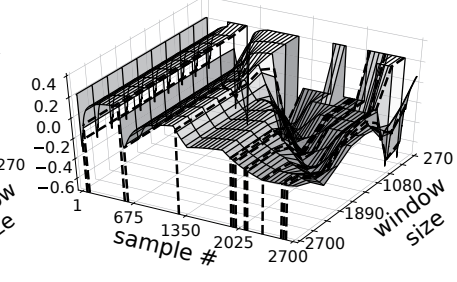

(b) $H A T$

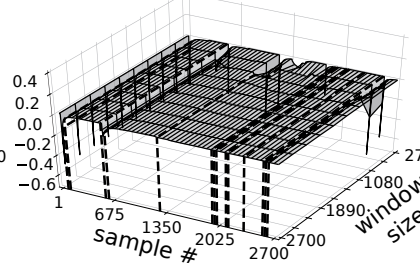

(c) $A R F$

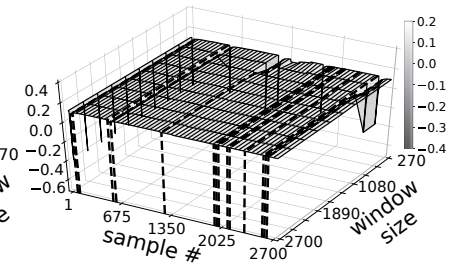

(d) $S G D$

Figure 3: Prequential AUC Evaluation. Diagrams show the dependency of the Prequential AUC results on the evaluation window size, for each attack type. Values above zero show when the stream algorithm performance exceeds the batch result. Concept drifts are marked with dashed lines.

span. Note that the frequency of changes significantly increases in the last third of the dataset, with more than 10 changes detected in the last 4 days. It would be interesting to understand the reasons behind such changes, but this is out of scope for this paper. There are naturally multiple reasons for concept drifts, from modifications of the underlying characteristics of the prediction target to dynamic modifications in the classes-balance of the studied problem. Indeed, imbalanced datasets which do not maintain the inter-classes balance along time are a common phenomena resulting in concept drift in real data [16].

Concept drifts would be later on used to explain sudden shifts in algorithms' performance as depicted in Figs. 2 and 3. For example, when considering the ADWIN-based evaluations, the window size 


\begin{tabular}{|c|c|c|c|c|c|c|c|c|c|c|c|c|c|c|c|c|}
\hline \multirow{2}{*}{ attack type } & \multicolumn{2}{|c|}{$k-\mathbf{N N}$} & \multicolumn{2}{|c|}{$k$-NN-batch } & \multicolumn{2}{|c|}{ HAT } & \multicolumn{2}{|c|}{ HT-batch } & \multicolumn{2}{|c|}{ ARF } & \multicolumn{2}{|c|}{ RF-batch } & \multicolumn{2}{|c|}{ SGD } & \multicolumn{2}{|c|}{ SGD-batch } \\
\hline & AUC & $\mathrm{ACC}[\%]$ & AUC & ACC [\%] & AUC & ACC[\%] & AUC & $\mathrm{ACC}[\%]$ & AUC & ACC [\%] & AUC & $\mathrm{ACC}[\%]$ & AUC & ACC [\%] & AUC & $\mathrm{ACC}[\%]$ \\
\hline DDDSSYN & 0,96 & 63,5 & 0,94 & 86,9 & 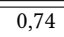 & 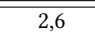 & 0,62 & 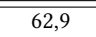 & 0,99 & 95,6 & 0,99 & 995,0 & 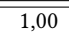 & 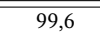 & 0,89 & 89,1 \\
\hline mptpla & 0,96 & 85,6 & 0,93 & 84,2 & 0,79 & 99,6 & 0,71 & 65,9 & 0,99 & 98,2 & 0,99 & 95,3 & 0,99 & 99,3 & 0,89 & 88,8 \\
\hline icmp_error & 0,97 & 64,7 & 0,93 & 89,5 & 0,71 & 0,4 & 0,50 & 76,4 & 0,99 & 94,3 & 0,99 & 95,4 & 0,99 & 99,5 & 0,87 & 91,4 \\
\hline ntscUDP & 0,98 & 61,8 & 0,96 & 91,3 & 0,73 & 93,7 & 0,78 & 75,1 & 0,99 & 95,5 & 1,00 & 97,0 & 0,99 & 99,5 & 0,90 & 92,6 \\
\hline ntscACK & 0,96 & 60,2 & 0,94 & 87,4 & 0,60 & 9,8 & 0,78 & 73,8 & 0,99 & 97,0 & 0,99 & 95,1 & 0,99 & 99,3 & 0,89 & 88,7 \\
\hline
\end{tabular}

Table 1: Benchmark results. ACC and AUC results for every analyzed attack type and model.

of the algorithm changes when concept drifts are detected, and thus could have an important impact on performance.

\subsection{Performance Evaluation - ADWIN}

We evaluate the performance of the algorithms in five binary classification variants - one for each attack type, resulting in five sets of results for each tested algorithm. Fig. 2 reports the performance results for each attack type, considering detection accuracy as performance metric, and using the batch-algorithms accuracy as baseline. The prequential 10-fold CV performance evaluation shows that both the Adaptive Random Forests (ARF) and the Stochastic Gradient Descent (SGD) models rapidly converge to the batch-based accuracy results, with minimum performance variations under concept drifts, and with a slightly better performance for the SGD model, this one even outperforming the batch-based performance.

On the other hand, both the $k$-NN and HAT models do not show any apparent convergence and results tend to oscillate around the batch algorithm baseline. In the case of HAT we can appreciate the correlation between the detected concept drifts and the performance variations of the model. Interestingly, the HAT model is the one achieving the highest accuracy of the four models (up to $40 \%$ improvement w.r.t. the baseline), but cannot maintain such performance constantly in time, with significant deterioration.

We here take the opportunity to flag, as reflected by the aforementioned results, that stream-based algorithms can largely outperform batch versions, at least during transient periods; for example, let us take the performance of the HAT algorithm. There is a general misconception around the stream-vs-batch performance comparison problem, and many tend to think that batch algorithms are always the best-case performance that a stream algorithm can attain; while this is generally true under stable learning scenarios, it is not under concept drifts, where irrelevant or no longer valid learning inputs can actually degrade the learning performance. In such scenarios, a stream-based approach using only the most recent set of measurements for training can largely improve over a batch containing all past measurements.

\subsection{Performance Evaluation - Prequential AUC}

We focus now on the prequential AUC evaluation. We test the dependency of the results on the sliding window size, by setting it to a fraction of the total dataset size: $\{10 \%, 20 \%, \ldots, 40 \%, \ldots, 100 \%\}$, i.e. $\{270,540, \ldots, 1080, \ldots, 2700\}$. Fig. 3 shows the prequential AUC values along time (number of samples) - considering the batch-based AUC values as baseline, for the different tested window sizes.

For all four models, and as expected, we can first observe how increasing the window size smooths performance variations along time. However, as observed before for the case of ADWIN, using smaller or more fine-tuned window sizes can translate into better performance; for example, the HAT model achieves a performance gain of up to $40 \%$ (w.r.t. the baseline) when using $10 \%$-dataset window size. Indeed, the window size allows to track long or short-term changes better, depending on the tuning.

As before, we see how both ARF and SGD are highly robust to concept drifts and converge for almost all window sizes; this also happens - to a lesser extent, for the $k$-NN model, which finds convergence and robustness for window size above $40 \%$ of the dataset size. Note how HAT performance also starts converging for longer window sizes, but with an important performance degradation for some attack types, directly implying that keeping pass history under concept drifts might negatively impact results.

To conclude the study, and to serve as benchmark, Tab. 1 reports the accuracy (ACC) and AUC results for every analyzed attack type and model. For stream algorithms we report the last evaluation value, with AUC corresponding to the largest windows size (i.e., 2700), for convergence purposes. This evaluation approach explains the poor performance values reported for HAT. As already mentioned, the benchmark shows the out-performance of both the ARF and SGD models, suggesting these are good candidates for stream-based network traffic monitoring and analysis.

\section{CONCLUSION}

In this paper, we have compared and analyzed the results from the most recent evaluation approaches for sequential data on commonly used batch-based machine learning algorithms and their corresponding stream-based extensions, for the specific problem of on-line network security and anomaly detection. Prequential 10-fold CV evaluation using ADWIN for dynamic window sizing makes a good choice for stream-based algorithm benchmarking in these applications, as it can better track transient changes and concept drifts. Similar results can be obtained by fine-tuning the window size, but requires additional calibration efforts. We have confirmed that both ARF and SGD models are better for the studied problem, specially in terms of robustness to concept drifts and convergence of results. The benchmark revealed that these algorithms achieve similar - and even better performance, than their corresponding batch-versions, but in a dynamic, stream-based scenario, with all the corresponding benefits for on-line traffic analysis. Our next steps include the integration of the MOA data streaming analytics into the Big-DAMA platform [26] using an Apache Flink distributed stream-processing framework [27], as well as analyzing the SAMOA framework for massive on-line data analytics. 


\section{REFERENCES}

[1] R. Fontugne, P. Borgnat, P. Abry, and K. Fukuda, "Mawilab: combining diverse anomaly detectors for automated anomaly labeling and performance benchmarking," in Proceedings of the 6th ACM CoNEXT Conference, 2010.

[2] V. Chandola, A. Banerjee, and V. Kumar, "Anomaly detection: A survey, ACM Comput. Surv., vol. 41, no. 3, pp. 15:1-15:58, Jul. 2009.

[3] M. Ahmed, A. Naser Mahmood, and J. Hu, "A survey of network anomaly detec tion techniques," f. Netw. Comput. Appl., vol. 60, no. C, pp. 19-31, Jan. 2016

[4] W. Zhang, Q. Yang, and Y. Geng, "A survey of anomaly detection methods in networks," in 2009 CNMT, Jan 2009, pp. 1-3.

[5] T. T. T. Nguyen and G. Armitage, "A survey of techniques for internet traffic classification using machine learning," IEEE Communications Surveys Tutorials, vol. 10 , no. 4 , pp. 56-76, Fourth 2008

[6] J. Vanerio and P. Casas, "Ensemble-learning approaches for network security and anomaly detection," in Proceedings of the ACM SIGCOMM Big-DAMA Workshop. New York, NY, USA: ACM, 2017, pp. 1-6.

[7] P. Casas, F. Soro, J. Vanerio, G. Settanni, and A. D'Alconzo, "Network security and anomaly detection with big-dama, a big data analytics framework," in 2017 IEEE 6th CloudNet Conference, Sept 2017, pp. 1-7.

[8] P. Casas, J. Vanerio, and K. Fukuda, "Gml learning, a generic machine learning model for network measurements analysis," in 2017 13th International Conference on Network and Service Management (CNSM), Nov 2017, pp. 1-9.

[9] P. Casas and J. Vanerio, "Super learning for anomaly detection in cellular networks," in 2017 IEEE 13th WiMob Conference, Oct 2017, pp. 1-8.

[10] V. Carela-Español, P. Barlet-Ros, A. Bifet, and K. Fukuda, "A streaming flowbased technique for traffic classification applied to $12+1$ years of internet traffic," Telecommunication Systems, vol. 63, no. 2, pp. 191-204, 2016.

[11] P. M. Domingos and G. Hulten, "Catching up with the data: Research issues in mining data streams." in $D M K D, 2001$.

[12] M. Stonebraker, U. Çetintemel, and S. Zdonik, "The 8 requirements of real-time stream processing," ACM Sigmod Record, vol. 34, no. 4, pp. 42-47, 2005.

[13] G. Hulten, P. Domingos, and L. Spencer, Mining massive data streams. University of Washington, 2005.

[14] J. Gama, R. Sebastião, and P. P. Rodrigues, "Issues in evaluation of stream learning algorithms," in Proceedings of the 15th ACM SIGKDD Conference. ACM, 2009, pp. 329-338.

[15] --, "On evaluating stream learning algorithms," Machine learning, vol. 90, no. 3, pp. 317-346, 2013.

[16] T. R. Hoens, R. Polikar, and N. V. Chawla, "Learning from streaming data with concept drift and imbalance: an overview," Progress in Artificial Intelligence, vol. 1, no. 1 , pp. 89-101, 2012.

[17] G. Hulten and P. Domingos, "Vfml-a toolkit for mining high-speed time-changing data streams," Software toolkit, p. 51, 2003.

[18] X. Meng, J. Bradley, B. Yavuz, E. Sparks, S. Venkataraman, D. Liu, J. Freeman, D. Tsai, M. Amde, S. Owen et al., "Mllib: Machine learning in apache spark," The fournal of Machine Learning Research, vol. 17, no. 1, pp. 1235-1241, 2016.

[19] A. Bifet, G. Holmes, R. Kirkby, and B. Pfahringer, "Moa: Massive online analysis," Journal of Machine Learning Research, vol. 11, no. May, pp. 1601-1604, 2010.

[20] O. Rittho, R. Klinkenberg, S. Fischer, I. Mierswa, and S. Felske, "Yale: Yet another learning environment," in LLWA 01-Tagungsband der GI-Workshop-Woche LernenLehren-Wissen-Adaptivität, no. 763. Citeseer, 2001, pp. 84-92.

[21] G. D. F. Morales and A. Bifet, "Samoa: scalable advanced massive online analysis." Journal of Machine Learning Research, vol. 16, no. 1, pp. 149-153, 2015.

[22] A. Bifet and R. Gavalda, "Learning from time-changing data with adaptive windowing," in Proceedings of the 2007 SIAM Conference, 2007, pp. 443-448.

[23] A. Bifet, G. de Francisci Morales, J. Read, G. Holmes, and B. Pfahringer, "Efficient online evaluation of big data stream classifiers," in Proceedings of the 21th ACM SIGKDD Conference. ACM, 2015, pp. 59-68.

[24] D. Brzezinski and J. Stefanowski, "Prequential auc: properties of the area under the roc curve for data streams with concept drift," Knowledge and Information Systems, vol. 52, no. 2, pp. 531-562, 2017.

[25] J. Gama, I. Žliobaitè, A. Bifet, M. Pechenizkiy, and A. Bouchachia, "A survey on concept drift adaptation," ACM CSUR, vol. 46, no. 4, p. 44, 2014

[26] P. Casas, A. D'Alconzo, T. Zseby, and M. Mellia, "Big-dama: big data analytic for network traffic monitoring and analysis," in Proceedings of the 2016 ACM SIGCOMM LANCOMM Workshop. ACM, 2016, pp. 1-3.

[27] P. Carbone, A. Katsifodimos, S. Ewen, V. Markl, S. Haridi, and K. Tzoumas, "Apache flink: Stream and batch processing in a single engine," Bulletin of the IEEE Computer Society TC on Data Engineering, vol. 36, no. 4, 2015. 